\title{
Kaon form factor in holographic QCD
}

\author{
Zainul Abidin ${ }^{1, *}$ and Parada T. P. Hutauruk ${ }^{2, \dagger}$ \\ ${ }^{1}$ Sekolah Tinggi Keguruan dan Ilmu Pendidikan Surya, Tangerang, Jawa Barat 15115, Indonesia \\ ${ }^{2}$ Asia Pacific Center for Theoretical Physics, Pohang, Gyeongbuk 37673, Korea
}

(Received 23 May 2019; published 19 September 2019)

\begin{abstract}
The kaon form factor in the spacelike region is calculated using a holographic QCD model with the "bottom-up" approach. We found that our result for the kaon form factor in low $Q^{2}$ has a remarkable agreement with the existing data, where $Q^{2}$ is the four-momentum transfer squared. The charge radius of the kaon as well as the kaon decay constant are found to be in good agreement with the experiment data. We then predict the kaon form factor in the asymptotic region (larger $Q^{2}$ ) showing $1 / Q^{2}$ behavior, which is consistent with the perturbative QCD prediction.
\end{abstract}

DOI: 10.1103/PhysRevD.100.054026

\section{INTRODUCTION}

A theory of quantum chromodynamics (QCD), which is a non-Abelian gauge theory, is believed so far as a correct theory of hadrons, where hadrons are the composite particles made of quarks and gluons [1]. QCD has the essential features, namely, confinement and chiral symmetry breaking $[1,2]$. However, the form factor, which is one of the nonperturbative quantities, is very difficult to compute directly from QCD. Several theoretical and phenomenological models [3-7] as well as a lattice QCD calculation [8] have been used to calculate this nonperturbative quantity of QCD.

Apart from those models, during the past few years, holographic QCD models, which are the complementary model of QCD, have also been applied to describe the structure of hadrons, namely, meson [9-14] and nucleon [14] form factors as well as charmed meson [15], in order to gain a deep understanding of the structure of hadrons, from a different substantially point of view. Surprisingly, these holographic models work well in predicting other hadron observables, namely, the decay constant and mass spectrum. Also, one can argue that QCD approximately behaves as a conformal over a particular kinematic region $[13,16]$. Those holographic QCD models are able to preserve confinement $[11,17,18]$ and chiral symmetry breaking $[12,19-21]$, which is in many ways similar to the main

\footnotetext{
zainul.abidin@stkipsurya.ac.id

parada.hutauruk@apctp.org
}

Published by the American Physical Society under the terms of the Creative Commons Attribution 4.0 International license. Further distribution of this work must maintain attribution to the author(s) and the published article's title, journal citation, and DOI. Funded by SCOAP ${ }^{3}$. properties of QCD in low energy, after a few years since the holographic model was proposed $[22,23]$.

The original AdS/CFT correspondence [22] has been first used to connect a strongly coupled $4 \mathrm{D}$ conformal theory for large $N_{c}$, where $N_{c}$ is the color number, and a weakly coupled gravity theory on AdS space. It then has been reconstructed starting from QCD and its 5D gravity dual theory to reproduce the properties of QCD [16,24-26].

However, among those holographic models with various approaches [9-15,17-23], only a few models have been used to calculate the kaon form factor in a holographic QCD model with different approaches [15]. $K^{+}(u \bar{s})$ is a very interesting object, because it consists of a strange quark, beside an up quark, where the mass of the strange quark is heavier than the $u$ quark. Experimentally, the existing data on the kaon form factor are very poor in higher $Q^{2}$, and only old data for low $Q^{2}$ are available [27], where $Q^{2}$ is the four-momentum transfer squared. In the future, experiments will measure the kaon form factor in higher $Q^{2}$ $[28,29]$. It would be interesting to see how our complementary model, which is inspired by this AdS/CFT correspondence, predicts the kaon form factor in higher $Q^{2}$. This work may pave the way to understand the strange quark properties as well as the strange quark form factor.

In the present paper, we calculate the kaon form factor in holographic QCD, which is a complementary approach of QCD. In this work, we adopt a "bottom-up" approach of the AdS/CFT correspondence, instead of a "top-down" approach, where we employ the properties of QCD to construct its 5D gravity dual theory as performed in Refs. [24,25,30]. We begin to describe the AdS/CFT correspondence formalism, describing a correspondence between 4D operators $\mathcal{O}(x)$ and fields in the 5D bulk $\phi(x, z)$. We then calculate the kaon form factor in holographic QCD. We find the result on the kaon form factor is in good agreement compared to the existing data in low $Q^{2}$ 
[27]. We then predict the kaon form factor in higher $Q^{2}$. Experimentally, the experimental data are really poor in higher $Q^{2}$. We find that the kaon form factor in higher $Q^{2}$ is consistent with the perturbation QCD prediction [31]. Next, we calculate the charge radius of the kaon in holography. We find that our result on the charge radius is an excellent agreement with the data [27] as well as the Particle Data Group (PDG) [32].

This paper is organized as follows. In Sec. II, we briefly review the AdS/CFT correspondence, two- and three-point functions, and how to extract the form factor of the kaon from holography QCD in Sec. III. In Sec. IV, we present the calculation of the charge radius of the kaon. In Sec. V, numerical results are presented and their implications are discussed. Section VI is devoted to a summary.

\section{FORMALISM}

\section{A. The AdS/QCD correspondence}

In this section, we briefly review the calculation of the vacuum expectation values of the operators based on a generating function $Z_{4 \mathrm{D}}$ in the $4 \mathrm{D}$ space, which is defined by

$$
Z_{4 \mathrm{D}}\left[\phi^{0}\right]=\left\langle\exp \left(i S_{4 \mathrm{D}}+i \int d^{4} x \mathcal{O}(x) \phi^{0}(x)\right)\right\rangle
$$

where $S_{4 \mathrm{D}}$ is the action for the $4 \mathrm{D}$ theory and $\phi^{0}$ is a source function together with a specific operator $\mathcal{O}(x)$, which corresponds to the expectation value. It then can be written by

$$
\left\langle 0\left|\mathcal{T} \mathcal{O}\left(x_{1}\right) \ldots \mathcal{O}\left(x_{n}\right)\right| 0\right\rangle=\frac{(-i)^{n} \delta Z_{4 \mathrm{D}}}{\delta \phi^{0}\left(x_{1}\right) \ldots \phi^{0}\left(x_{n}\right)} .
$$

The following AdS/CFT correspondence provides the equivalence between the generating functional of the connected correlation for the 4D theory and the effective partition function for the 5D theory:

$$
Z_{4 \mathrm{D}}\left[\phi^{0}\right]=\exp \left(i S_{5 \mathrm{D}}\left(\phi_{c l}\right)\right),
$$

where $\phi_{c l}$ is a solution of the 5D equation of motion with a boundary, as defined in Eq. (5).

We consider only the tree-level diagram on the 5D theory, and we choose the following metric for the $5 \mathrm{D}$ space-time:

$d s^{2}=g_{M N} d x^{M} d x^{N}=\frac{1}{z^{2}}\left(\eta_{\mu \nu} d x^{\mu} d x^{\nu}-d z^{2}\right), \quad \varepsilon<z<z_{0}$,

where $x$ is the 4D space-time coordinate, $\eta_{\mu \nu}=\operatorname{diag}(1,-1$, $-1,-1)$ is the flat space metric, and $z$ is the fifth coordinate, which corresponds to the energy scale $(Q \sim 1 / z)$. We set $z=\varepsilon \rightarrow 0$ for the ultraviolet boundary of the $5 \mathrm{D}$ space that relates with the UV limit of QCD, and the hard-wall cutoff at $z=z_{0}=1 / \Lambda_{\mathrm{QCD}}$ is the infrared boundary, which is used for the conformal symmetry breaking of QCD.

The UV boundary value of the 5D field is the source of the corresponding 4D operator $\mathcal{O}$. One can write the classical solution of the 5D field as

$$
\phi_{c l}(x, z)=\phi(x, z) \phi^{0}(x)
$$

The value of $\phi(x, \varepsilon) \rightarrow 1$ (or, in general, it goes to $\varepsilon^{\Delta}$ ). Hence, $\phi^{0}(x)$ is identified as the UV-boundary value of the $\phi_{c l}(x, z)$ field.

\section{B. The 5D AdS model}

The action in 5D theory is written as

$$
S_{5 \mathrm{D}}=\int d^{5} x \sqrt{g} \operatorname{Tr}\left\{|D X|^{2}+3|X|^{2}-\frac{1}{4 g_{5}^{2}}\left(F_{L}^{2}+F_{R}^{2}\right)\right\},
$$

where $g=\left|\operatorname{det} g_{M N}\right|$ is the determinant of metric tensor, $g_{5}$ is a gauge coupling parameter, which is fixed by the QCD operator product expansion, and the bifundamental scalar field $X$ in Eq. (6) is expressed by

$X(x, z)=\exp \left(i \pi^{a}(x, z) t^{a}\right) X^{0}(z) \exp \left(-i \pi^{a}(x, z) t^{a}\right)$,

where $t^{a}=\sigma^{a} / 2$ are the $\mathrm{SU}(3)$ generators with $\operatorname{Tr}\left[t^{a} t^{b}\right]=\delta^{a b} / 2$, where $\sigma^{a}$ are the Pauli matrices. The covariant derivative is defined as

$$
D_{M} X=\partial_{M} X-i L_{M} X+i X R_{M},
$$

where the $5 \mathrm{D}$ space-time is denoted by the lowercase index of $M=(\mu, z)$ and $F_{M N}^{L}$ is written as

$$
F_{M N}^{L}=\partial_{M} L_{N}-\partial_{N} L_{M}-i\left[L_{M}, L_{N}\right],
$$

Analogously for $F_{M N}^{R}$.

The $L$ and the $R$ fields can be written as vector field $V$ and the axial-vector field $A$ :

$$
\begin{aligned}
& L_{M}=V_{M}+A_{M}, \\
& R_{M}=V_{M}-A_{M} .
\end{aligned}
$$

In this work, we consider the following $4 \mathrm{D}$ operators that are defined by the current operators $J_{L \mu}^{a}=\bar{\psi}_{q L} \gamma_{\mu} t^{a} \psi_{q L}$ and $J_{R \mu}^{a}=\bar{\psi}_{q R} \gamma_{\mu} t^{a} \psi_{q R}$ that correspond to the gauge fields $L_{\mu}^{a}$ and $R_{\mu}^{a}$ in the 5D theory, respectively. The operator of $\bar{\psi}_{q_{R}} \psi_{q_{L}}$ corresponds to a bifundamental scalar field $X^{a}$ in Eq. (7), where the index $a=1,2, \ldots, 8$ for $\mathrm{SU}(3)$ flavor symmetry and index $\mu=0,1,2,3$ for the space-time. 
Note that the gauge invariance in the 5D theory is related with the global current conservation in the 4D theory.

\section{Two-point functions}

Here we consider only the scalar parts of the action $X^{0}(z)$; up to second order, it gives

$$
S_{\text {scalar }}=\int d^{5} x \sqrt{g} \operatorname{Tr}\left(g^{M N} \partial_{M} X^{0} \partial_{N} X^{0}+3\left|X^{0}\right|^{2}\right),
$$

where $g^{M N}$ is defined in Eq. (4), which is the nontrivial 5D metric.

The UV boundary of the scalar field $X^{0}$ is proportional to the quark mass matrix $M$, which can be considered as the source for the operator of $\bar{\psi}_{R} \psi_{L}$. Solving the equation of motion for the scalar field, it then gives

$$
X^{0}(z)=a_{1} z+a_{3} z^{3}
$$

where $a_{1}$ is defined as in Ref. [30] by

$$
a_{1}=\frac{1}{2} M \mathbb{1}=\frac{1}{2}\left(\begin{array}{lll}
m_{q} & & \\
& m_{q} & \\
& & m_{s}
\end{array}\right)
$$

where we consider the $\mathrm{SU}(2)$ isospin symmetry where the mass for the up and down quarks are identical.

Using the AdS/CFT correspondence, we then calculate the quark condensate $\langle\bar{\psi} \psi\rangle=\Sigma$ by performing a functional derivative of the action in Eq. (12), evaluated on the classical solution, over $\delta M$ and identify

$$
a_{3}=\frac{1}{2} \Sigma \mathbb{1}=\frac{1}{2}\left(\begin{array}{ccc}
\sigma_{q} & & \\
& \sigma_{q} & \\
& & \sigma_{s}
\end{array}\right) \text {. }
$$

We also assume that $\sigma_{q}=\sigma_{s}=\sigma$ and define $v_{q}(z)=$ $m_{q} z+\sigma z^{3}$ and $v_{s}(z)=m_{s} z+\sigma z^{3}$.

\section{Transverse vector}

We now consider only vector parts of the action up to second order. It gives

$$
\begin{aligned}
S_{\text {vector }}= & \int d^{5} x \sum_{a=4}^{8} \frac{1}{4 g_{5}^{2} z}\left(-\left(\partial_{M} V_{N}^{a}-\partial_{N} V_{M}^{a}\right)^{2}\right. \\
& \left.+2 \alpha^{a}(z)\left(V_{M}^{a}\right)^{2}\right) .
\end{aligned}
$$

A contraction over 5D metric $\eta_{M L}$ is implied. We then define

$$
\alpha^{a}(z)= \begin{cases}0 & a=1,2,3 \\ g_{5}^{2}\left(v_{s}-v_{q}\right)^{2} /\left(4 z^{2}\right) & a=4,5,6,7 \\ 0 & a=8\end{cases}
$$

We have gauge choice to set $V_{z}^{a}=0$ except for $a=4,5$, 6,7 because of the nonzero ("mass term") of the second term in the action of Eq. (16). The equation of motion for the 4D Fourier transform of the transverse part of the gauge field $V_{\perp, \mu}^{a}(q, z)$ is written as

$$
\left(\partial_{z} \frac{1}{z} \partial_{z}+\frac{q^{2}-\alpha^{a}}{z}\right) V_{\perp, \mu}^{a}(q, z)=0
$$

where $\alpha^{a}=g_{5}^{2}\left(m_{s}-m_{q}\right)^{2} / 4$ when $\sigma_{s}=\sigma_{q}$ for $a=4,5$, $6,7$.

One writes the transverse part of the vector field as $V_{\perp, \mu}^{a}(q, z)=V_{\perp, \mu}^{0, a}(q) V^{a}(q, z)$ with the so-called bulk-toboundary propagator $V^{a}(q, z)$, which is normalized to $V^{a}(q, \varepsilon)=1$ at the boundary condition $z=0$, and $V_{\perp, \mu}^{0, a}(q)$ is the Fourier transform of the source of the vector current $J_{V, \mu}^{a}=\bar{\psi}_{q_{v}} \gamma_{\mu} t^{a} \psi_{q_{v}}$ at the UV boundary $z=\varepsilon$. We also impose a Neumann boundary condition $\partial_{z} V\left(q^{2}, z_{0}\right)=0$. The solution for the bulk-to-boundary propagator is written as

$$
V^{a}\left(q^{2}, z\right)=\frac{\pi}{2} \tilde{q} z\left(\frac{Y_{0}\left(\tilde{q} z_{0}\right)}{J_{0}\left(\tilde{q} z_{0}\right)} J_{1}(\tilde{q} z)-Y_{1}(\tilde{q} z)\right),
$$

where $\tilde{q}^{2}=q^{2}-\alpha^{a}$ and $Y_{1}(x)$ and $J_{1}(x)$ are the Bessel functions, respectively.

For spacelike four-momentum transferred $q^{2}=-Q^{2}<0$, the solution in Eq. (19) can be written as

$$
V^{a}\left(Q^{2}, z\right)=\tilde{Q} z\left(\frac{K_{0}\left(\tilde{Q} z_{0}\right)}{I_{0}\left(\tilde{Q} z_{0}\right)} I_{1}(\tilde{Q} z)+K_{1}(\tilde{Q} z)\right)
$$

where $\tilde{Q}=\sqrt{Q^{2}+\alpha^{a}}$ and $K_{1}(x)$ and $I_{1}(x)$ are the modified Bessel functions, respectively.

The action on the solution in Eq. (20) is evaluated with applying transverse projector $\eta^{\mu \nu} \rightarrow\left(\eta^{\mu \nu}-\frac{q^{\mu} q^{\nu}}{q^{2}}\right)=P_{T}^{\mu \nu}$, since $\partial_{\mu} V_{\perp}^{a, \mu}=0$, one has the form

$$
S_{\text {vector }}=-\frac{1}{2 g_{5}^{2}} \int \frac{d^{4} q}{(2 \pi)^{4}} V_{\mu}^{0, a}(q) V_{\nu}^{0, a}(q) P_{T}^{\mu \nu} \frac{\partial_{z} V^{a}\left(q^{2}, \varepsilon\right)}{z} .
$$

After solving the differential part, by the AdS/CFT correspondence, we obtain the current-current two-point functions 


$$
\left\langle 0\left|\mathcal{T} J_{\perp}^{a, \mu}(x) J_{\perp}^{b, \nu}(y)\right| 0\right\rangle=\frac{i \delta^{2} S_{5 \mathrm{D}}}{i^{2} \delta V_{\perp, \mu}^{0, a}(x) \delta V_{\perp, \nu}^{0, b}(y)},
$$

where

$$
V_{\mu}^{0, a}(q)=\int d^{4} x e^{i q x} V_{\mu}^{0, a}(x)
$$

and this leads to

$$
\begin{gathered}
i \int d^{4} x e^{i q x}\left\langle 0\left|\mathcal{T} J_{\perp}^{a, \mu}(x) J_{\perp}^{b, \nu}(0)\right| 0\right\rangle \\
=-\frac{1}{g_{5}^{2}} P_{T}^{\mu \nu} \delta^{a b} \frac{\partial_{z} V^{a}\left(q^{2}, \varepsilon\right)}{z},
\end{gathered}
$$

where $\mathcal{T}$ is the time-ordering operator.

The bulk-to-boundary propagator can be written as

$$
V^{a}\left(q^{2}, z\right)=\sum_{n=0}^{\infty} c_{n}^{a}\left(q^{2}\right) \psi_{n}(z)
$$

where the wave function of $\psi_{n}$ satisfies the eigenvalue equation

$$
\left(\partial_{z} \frac{1}{z} \partial_{z}+\frac{\left(\left(M_{V, n}^{a}\right)^{2}-\alpha^{a}\right)}{z}\right) \psi_{n}^{a}(z)=0,
$$

which is normalized as

$$
\int d z \frac{1}{z} \psi_{n}^{a} \psi_{m}^{a}=\delta_{m n}
$$

with boundary condition $\psi_{n}^{a}(\varepsilon)=0=\partial_{z} \psi_{n}^{a}\left(z_{0}\right)$, and the solution is

$$
\psi_{n}^{a}(z)=\frac{\sqrt{2} z J_{1}\left(z \sqrt{\left(M_{V, n}^{a}\right)^{2}-\alpha^{a}}\right)}{z_{0} J_{1}\left(z_{0} \sqrt{\left(M_{V, n}^{a}\right)^{2}-\alpha^{a}}\right)},
$$

where the eigenvalues of $M_{V, n}^{a}$ (Kaluza-Klein tower of the mass of the vector mesons: $\rho$ meson for $a=1,2,3, K^{*}$ meson for $a=4,5,6,7$, and $\omega^{0}$ meson for $a=8$ ) are obtained from $J_{0}\left(z_{0} \sqrt{\left(M_{V, n}^{a}\right)^{2}-\alpha^{a}}\right)=0$.

Using Eqs. (18), (25), and (26), we obtain

$$
c_{n}^{a}\left(q^{2}\right)=-\frac{\frac{1}{\varepsilon} \partial_{z} \psi_{n}(\varepsilon)}{q^{2}-\left(M_{V, n}^{a}\right)^{2}} .
$$

Since

$$
\begin{aligned}
i \int & d^{4} x e^{i q x}\left\langle 0\left|\mathcal{T} J_{\perp}^{a, \mu}(x) J_{\perp}^{b, \nu}(0)\right| 0\right\rangle \\
= & \sum \frac{\left(f_{V, n}^{a}\right)^{2} \delta^{a b}}{q^{2}-\left(M_{V, n}^{a}\right)^{2}}\left(\eta^{\mu \nu}-\frac{q^{\mu} q^{\nu}}{q^{2}}\right) \\
& +(\text { nonpole terms }),
\end{aligned}
$$

where the definition of $f_{V, n}^{a}$ is given by the matrix element of current, $\left\langle 0\left|J_{\mu}^{a}\right| V_{n}^{c}(q, \lambda)\right\rangle=f_{V, n}^{a} \delta_{a c} \varepsilon_{\mu}(q, \lambda)$. We identify $f_{V, n}^{a}=\partial_{z} \psi_{n}^{a}(\varepsilon) / \varepsilon$. Also, the parameter $g_{5}^{2}=12 \pi^{2} / N_{c}=$ $4 \pi^{2}$ is fixed from the quark bubble diagram in the leading order, with $N_{c}=3$ the number of color.

\section{E. Axial-vector and pseudoscalar}

The action for the axial-vector and pseudoscalar sector parts up to second order is written as

$$
\begin{aligned}
S_{\text {axial }}= & \int d^{5} x \sum_{a=1}^{8} \frac{1}{4 g_{5}^{2} z}\left(-\left(\partial_{M} A_{N}^{a}-\partial_{N} A_{M}\right)^{2}\right. \\
& \left.+2 \beta^{a}(z)\left(\partial_{M} \pi^{a}-A_{M}^{a}\right)^{2}\right) .
\end{aligned}
$$

A contraction over 5D metric $\eta_{M L}$ is implied. We have gauge choice $A_{M}^{a} \rightarrow A_{M}^{a}-\partial_{M} \lambda^{a}$, and $\pi^{a} \rightarrow \pi^{a}-\lambda^{a}$ and $A_{z}^{a}=0$ are imposed. We define

$$
\beta^{a}(z)= \begin{cases}g_{5}^{2} v_{q}^{2} / z^{2} & a=1,2,3, \\ g_{5}^{2}\left(v_{q}+v_{s}\right)^{2} /\left(4 z^{2}\right) & a=4,5,6,7, \\ g_{5}^{2}\left(v_{q}^{2}+2 v_{s}^{2}\right) /\left(2 z^{2}\right) & a=8 .\end{cases}
$$

For the field $\phi$ that comes from the longitudinal part, we define $A_{\|, \mu}=\partial_{\mu} \phi^{a}$. We then write the Fourier transform of the fields in terms of the bulk-to-boundary propagators that gives

$$
\begin{aligned}
\phi^{a}(p, z) & =\phi^{a}\left(p^{2}, z\right) \phi^{0 a}(p)=\phi^{a}\left(p^{2}, z\right) \frac{i p^{\alpha}}{p^{2}} A_{\| \alpha}^{0 a}(p), \\
\pi^{a}(p, z) & =\pi^{a}\left(p^{2}, z\right) \frac{i p^{\alpha}}{p^{2}} A_{\| \alpha}^{0 a}(p), \\
A_{\perp \mu}^{b}(q, z) & =\mathcal{A}^{b}\left(q^{2}, z\right) A_{\perp \mu}^{0 b}(q),
\end{aligned}
$$

where $A_{\| \alpha}^{0 a}(p)$ is the Fourier transform of the source function of the 4D axial current operator $J_{A, \|}^{a, \alpha}$ and $A_{\perp \mu}^{0 b}(q)$ is the Fourier transform of the source function of the $4 \mathrm{D}$ axial current operator $J_{A, \perp}^{a, \alpha}$.

We obtain the coupled differential equations for the longitudinal part of the axial-vector and pseudoscalar fields as follows: 


$$
-q^{2} \partial_{z} \phi^{a}\left(q^{2}, z\right)+\beta^{a}(z) \partial_{z} \pi^{a}\left(q^{2}, z\right)=0
$$

$$
\partial_{z}\left(\frac{1}{z} \partial_{z} \phi^{a}\left(q^{2}, z\right)\right)-\frac{\beta^{a}(z)}{z}\left(\phi^{a}\left(q^{2}, z\right)-\pi^{a}\left(q^{2}, z\right)\right)=0,
$$

with the boundary conditions $\phi^{a}\left(q^{2}, \varepsilon\right)=0, \pi^{a}\left(q^{2}, \varepsilon\right)=-1$, and $\partial_{z} \phi^{a}\left(q^{2}, z_{0}\right)=0=\partial_{z} \pi^{a}\left(q^{2}, z_{0}\right)$. The expression for the transverse part of the axial-vector field is analogous to the vector field. It then gives

$$
\left(\partial_{z} \frac{1}{z} \partial_{z}+\frac{q^{2}-\beta^{a}(z)}{z}\right) A_{\perp}^{a}\left(q^{2}, z\right)=0 .
$$

We then substitute the coupled differential in Eqs. (34) and (35) into a second-order equation, and we obtain

$$
\partial_{z}\left(\frac{z}{\beta^{a}(z)} \partial_{z} y^{a}\left(q^{2}, z\right)\right)+z\left(\frac{q^{2}}{\beta^{a}(z)}-1\right) y^{a}\left(q^{2}, z\right)=0,
$$

where $y^{a}\left(q^{2}, z\right)=\partial_{z} \phi^{a}\left(q^{2}, z\right) / z$. In this form, the boundary condition is $y\left(q^{2}, z_{0}\right)=0$ and $\varepsilon \partial_{z} y^{a}\left(q^{2}, \varepsilon\right) / \beta^{a}(\varepsilon)=1$. We then have the solution as

$$
y^{a}\left(q^{2}, z\right)=\sum \frac{\left(M_{\pi, n}^{a}\right)^{2} y_{n}^{a}(\varepsilon) y_{n}^{a}(z)}{q^{2}-\left(M_{\pi, n}^{a}\right)^{2}}
$$

where $y_{n}^{a}(z)$ is a normalized solution of the eigenvalue in Eq. (37) with $q^{2}=M_{\pi, n}^{a}$, boundary conditions $y_{n}\left(z_{0}\right)=0$, and $\varepsilon \partial_{z} y_{n}^{a}(\varepsilon) / \beta(\varepsilon)=0$. The normalization is

$$
\int \frac{z}{\beta^{a}(z)} y_{n}^{a}(z) y_{m}^{a}(z)=\frac{\delta_{m n}}{\left(M_{\pi, n}^{a}\right)^{2}} .
$$

The eigenvalues of $\left(M_{\pi, m}^{a}\right)^{2}$ are the Kaluza-Klein (KK) masses for the pseudoscalar mesons: the pions for $a=1,2$, 3 , kaons for $a=4,5,6,7$, and $\eta^{0}$ 's for $a=8$. The eigenvalues are obtained from the transverse part of the axial vector in Eq. (36), giving us the KK mass of the $a_{1}$ and $K_{1}$ mesons.

As noted above, for the vector sector, we do not have the freedom to set $V_{z}^{a}=0$, for $a=4,5,6,7$. However, if we define $V_{z}^{a}=-\partial_{z} \tilde{\pi}^{a}, V_{\|, \mu}^{a}=\partial_{\mu}\left(\tilde{\phi}^{a}-\tilde{\pi}^{a}\right)$, we obtain analogous equations as in Eqs. (34) and (35) with $\alpha^{a}(z)$ in place of $\beta^{a}(z)$. We may proceed as above to obtain the eigenvalues of the KK mass of the scalar mesons $K_{0}^{*}$.
A current-current correlator for the axial sector is written as

$$
\begin{aligned}
& i \int_{x} e^{i q x}\left\langle 0\left|\mathcal{T} J_{A \perp}^{a \mu}(x) J_{A \perp}^{b \nu}(0)\right| 0\right\rangle=-P_{T}^{\mu \nu} \delta^{a b} \frac{\partial_{z} A_{\perp}^{a}\left(q^{2}, \varepsilon\right)}{g_{5}^{2} \varepsilon} \\
& i \int_{x} e^{i q x}\left\langle 0\left|\mathcal{T} J_{A \|}^{a \mu}(x) J_{A \|}^{b \nu}(0)\right| 0\right\rangle=-P_{L}^{\mu \nu} \delta^{a b} \frac{\partial_{z} \phi^{a}\left(q^{2}, \varepsilon\right)}{g_{5}^{2} \varepsilon}
\end{aligned}
$$

where $P_{L}^{\mu \nu}=q^{\mu} q^{\nu} / q^{2}$. Using the completeness relation $\sum_{n} \int \frac{d^{3} q}{(2 \pi)^{3} 2 q^{0}}|n(q)\rangle\langle n(q)|=1$ into the correlators in Eq. (40), then multiplying $q^{2}-m_{n}^{2}$, and taking a limit $q^{2} \rightarrow m_{n}^{2}$, one identifies the decay constant of the pseudoscalar mesons from AdS/QCD correspondence:

$$
f_{A, n}^{a}=-\frac{y_{n}^{a}(\varepsilon)}{g_{5}}=-\frac{\partial_{z} \phi_{n}^{a}(\varepsilon)}{g_{5} \varepsilon},
$$

where the decay constants are defined by

$$
\left\langle 0\left|J_{A \mu \|}^{a}(0)\right| \pi_{n}^{b}(q)\right\rangle=i f_{A, n}^{a} q_{\mu} \delta^{a b},
$$

where the states of $\left|\pi_{n}^{b}(q)\right\rangle$ are also considered for the pions $(b=1,2,3)$ as well as the kaons $(b=4,5,6,7)$.

\section{KAON ELECTROMAGNETIC FORM FACTOR}

The electromagnetic form factors of the pion and kaon are presented in this section. The relevant parts of the action are

$$
\begin{aligned}
S_{A_{\|} V_{\perp} A_{\|}} & =\int d^{5} x\left(\frac{1}{g_{5}^{2} z} \partial^{\mu} \phi^{a} \partial_{\mu} V_{\nu}^{b} \partial^{\nu} \phi^{c} f^{a b c}\right. \\
& +\frac{1}{z^{3}}\left(\partial^{\mu} \pi^{a}-\partial^{\mu} \phi^{a}\right) V_{\mu}^{b} \pi^{c} g^{a b c} \\
& \left.+\frac{1}{z^{3}}\left(-\frac{1}{2} \partial^{\mu}\left(\pi^{a} \pi^{c}\right)+\partial^{\mu} \phi^{a} \pi^{c}\right) V_{\mu}^{b} h^{a b c}\right),
\end{aligned}
$$

where the first term in Eq. (43) that contains $f^{a b c}$ arises from the gauge part of the original action and other terms come from the chiral part. We then define

$$
\begin{aligned}
& g^{a b c}=-2 i \operatorname{Tr}\left\{t^{a}, X_{0}\right\}\left[t^{b},\left\{t^{c}, X_{0}\right\}\right], \\
& h^{a b c}=-2 i \operatorname{Tr}\left[t^{b}, X_{0}\right]\left\{t^{a},\left\{t^{c}, X_{0}\right\}\right\} .
\end{aligned}
$$

If $g^{a b c}$ and $h^{a b c}$ in Eq. (44) do not have $a, b$, or $c$, which do not equal " 8 ," it then gives

$$
\begin{aligned}
& g^{a b c}=f^{a b c} v_{a} v_{c}, \\
& h^{a b c}=f^{a b c}\left(v_{c}-v_{a}\right) v_{c},
\end{aligned}
$$

where, for $X_{0}=\frac{1}{2} c_{0}+c_{8} t^{8}$, the $v_{a}$ is defined as 


$$
v_{a}=c_{0}+c_{8} d^{a a 8}=\left\{\begin{array}{cl}
v_{q}, & a=1,2,3, \\
\frac{1}{2}\left(v_{q}+v_{s}\right), & a=4,5,6,7,
\end{array}\right.
$$

where $f^{a b c}$ and $d^{a b c}$ are the structure constants of the SU(3) algebra.

For three-point functions, we calculate three current operators by taking the functional derivative of Eq. (43). One has the form

$$
\left\langle 0\left|\mathcal{T} J_{A \|}^{a, \alpha}(x) J_{\perp}^{\mu}(y) J_{A \|}^{c, \beta}(w)\right| 0\right\rangle=\frac{i \delta S_{A_{\|} V_{\perp} A_{\|}}}{i^{3} \delta A_{\| \alpha}^{0 a}(x) \delta V_{\perp \mu}^{0 b}(y) \delta A_{\| \beta}^{0 c}(w)} .
$$

From Eq. (47), we then extract the form factor using the following matrix element:

$$
\begin{aligned}
& -f_{n}^{a *} f_{m}^{b} p^{\beta} k^{\alpha}\left\langle\pi_{n}^{a}(p)\left|J_{\perp}^{b, \mu}\right| \pi_{m}^{c}(k)\right\rangle(2 \pi)^{4} \delta^{4}(p-q-k) \\
& =\lim _{\substack{p^{2} \rightarrow\left(M_{\pi n}^{a}\right)^{2} \\
k^{2} \rightarrow\left(M_{\pi m}^{c}\right)^{2}}}\left(p^{2}-\left(M_{\pi n}^{a}\right)^{2}\right)\left(k^{2}-\left(M_{\pi m}^{c}\right)^{2}\right) \\
& \quad \times \int d^{4} x d^{4} y d^{4} w e^{i(p x-q y-k w)} \\
& \quad \times\left\langle 0\left|T J_{A \|}^{a \alpha}(x) J_{\perp}^{b \mu}(y) J_{A \|}^{c \beta}(w)\right| 0\right\rangle .
\end{aligned}
$$

We then obtain

$$
\begin{aligned}
& \left\langle\pi_{n}^{a}(p)\left|J_{\perp}^{b, \mu}\right| \pi_{m}^{c}(k)\right\rangle \\
& =i(p+k)^{\mu} \int d z V^{b}\left(q^{2}, z\right) \\
& \quad \times \frac{1}{z}\left(\left(\partial_{z} \phi_{n}^{a}\right)\left(\partial_{z} \phi_{m}^{c}\right)+\frac{g_{5}^{2} v_{a} v_{c}}{z^{2}}\left(\pi_{n}^{a}-\phi_{n}^{a}\right)\left(\pi_{m}^{c}-\phi_{m}^{c}\right)\right) f^{a b c} .
\end{aligned}
$$

For three quark flavors, the electromagnetic current operator is defined as

$$
J_{E M, \mu}=J_{\mu}^{3}+\frac{1}{\sqrt{3}} J_{\mu}^{8} .
$$

The current matrix element for the kaons $\left|K_{n}^{+}\right\rangle=$ $\left|\pi_{n}^{4}+i \pi_{n}^{5}\right\rangle$ is written as

$$
\left\langle K_{n}^{+}\left(p_{B}\right)\left|J_{E M, \mu}\right| K_{n}^{+}\left(p_{A}\right)\right\rangle=\left(p_{A}+p_{B}\right)^{\mu} F_{n n}^{K}\left(Q^{2}\right),
$$

where $Q^{2}=-q^{2}=-\left(p_{A}-p_{B}\right)^{2}$ and a final expression for the kaon form factor is obtained by

$$
\begin{aligned}
F_{n n}^{K}\left(Q^{2}\right)= & \int d z V^{3}\left(Q^{2}, z\right) \frac{1}{z}\left(\left(\partial_{z} \phi_{n}^{4}\right)\left(\partial_{z} \phi_{m}^{5}\right)\right. \\
& \left.+\frac{g_{5}^{2} v_{4}^{2}}{z^{2}}\left(\pi_{n}^{4}-\phi_{n}^{4}\right)\left(\pi_{m}^{5}-\phi_{m}^{5}\right)\right) .
\end{aligned}
$$

\section{KAON CHARGE RADIUS}

In this section, we present the charge radius of the kaon in low $Q^{2}$ as well as in higher $Q^{2}$. For doing so, we recall the kaon form factor in Eq. (52) that is

$$
F_{n n}^{K}\left(Q^{2}\right)=\int_{0}^{z_{0}} z V^{a}\left(Q^{2}, z\right) \rho_{n n}^{b}(z)
$$

where $a=1,2,3, b=4,5,6,7$, and $\rho_{n n}^{b}(z)$ is defined by

$$
\rho_{n n}^{b}(z)=\frac{\left(\partial_{z} \phi_{n}^{b}\right)^{2}}{z^{2}}+\frac{g_{5}^{2} v_{b}^{2}}{z^{4}}\left(\pi_{n}^{b}-\phi_{n}^{b}\right)^{2}
$$

In the limit of $Q \rightarrow 0$, the bulk-to-boundary propagator in Eq. (20) is written as

$$
V^{a}\left(Q^{2}, z\right)=1-\frac{Q^{2} z^{2}}{4}\left(1-2 \ln \left(\frac{z}{z_{0}}\right)\right) .
$$

Using the expansion of Eq. (20), we obtain the radius of the kaon as follows:

$$
\left\langle r_{K n}^{2}\right\rangle=-6 \frac{d F_{n n}^{K}\left(Q^{2}\right)}{d\left(Q^{2}\right)}=\int_{0}^{z_{0}} \frac{6}{4} z^{3}\left(1-2 \ln \left(\frac{z}{z_{0}}\right)\right) \rho_{n n}^{b}(z) .
$$

\section{NUMERICAL RESULTS}

Our numerical results for the kaon masses, decay constants, and kaon form factors are presented in this section. Following Ref. [13], we fix the parameter values of the hard-wall cutoff at $z_{0}=(322.5 \mathrm{MeV})^{-1}$, which is chosen to fit the lightest $\rho$ meson mass $M_{V, 1}^{a}=$ $775.5 \mathrm{MeV}$ for $a=1,2$, 3. Parameters $m_{q}$ and $\sigma_{q}$ is chosen to reproduce the pion mass and decay constant, respectively. Given the values of the pion mass $M_{\pi, 1}^{a}=$ 139.6 MeV and decay constant $f_{1}^{a}=92.4 \mathrm{MeV}$ for $a=1$, 2,3 , respectively, we obtain the light current quark mass $m_{q}=2.29 \mathrm{MeV}$ and condensate $\sigma_{q}=(328.3 \mathrm{MeV})^{3}$. We then fix $\sigma_{s}=\sigma_{q}$. The strange current quark mass $m_{s}=$ $51.96 \mathrm{MeV}$ is chosen to fit the kaon mass $M_{K, 1}^{a}=$ 495.7 MeV for $a=4,5,6,7$ (the masses for the $K^{+}$, $K^{-}, K^{0}$, and $\bar{K}^{0}$, respectively). We simply consider $m_{q}, m_{s}$, and $\sigma$ as model parameters, not the (realistic) physical values of the quark mass and quark condensate. For getting a better connection between the light current quark mass and condensate, we redefine the parameters by taking $m_{q} \rightarrow \sqrt{N_{c}} / 2 \pi$ and $\sigma \rightarrow 2 \pi / \sqrt{N_{c}}$ without modifying the above results for the two-point and three-point functions. With this redefinition, we obtain $m_{q}=8.31 \mathrm{MeV}$, $m_{s}=188.5 \mathrm{MeV}$, and $\sigma=(213.7 \mathrm{MeV})^{3}$.

Using the obtained parameters above, we determine the decay constant of the lightest $\mathrm{KK}$ of the kaons $f_{K^{+}}=$ $104 \mathrm{MeV}$, and the mass and decay constant of the $K_{0}^{*}$ are 


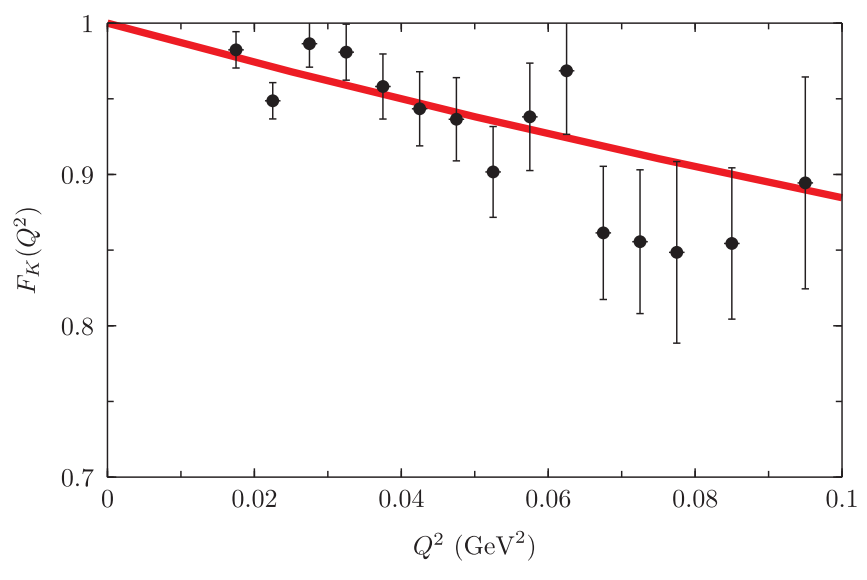

FIG. 1. The kaon form factor (solid line) compared to the existing data taken from Ref. [27].

$m_{K_{0}^{*}}=791 \mathrm{MeV}$ and $f_{K_{0}^{*}}=28 \mathrm{MeV}$, respectively. The decay constant of the $\rho$ meson $f_{\rho}^{1 / 2}=329 \mathrm{MeV}$. The mass and decay constant of the lightest KK of the vector mesons $K^{*}$ are $m_{K^{*}}=791 \mathrm{MeV}$ and $f_{K^{*}}^{1 / 2}=329 \mathrm{MeV}$, respectively. For the axial vector mesons, the mass and decay constant of the $a_{1}$ are $m_{a_{1}}=1366 \mathrm{MeV}$ and $f_{a_{1}}^{1 / 2}=489 \mathrm{MeV}$, respectively. For the $K_{1}$, we obtain $m_{K_{1}}=1458 \mathrm{MeV}$ and $f_{K_{1}}^{1 / 2}=511 \mathrm{MeV}$. The values of the decay constant and the mass of the kaon obtained are consistent with PDG [32].

Results for the kaon form factor are shown in Figs. 1-3. Figure 1 shows our prediction for the kaon form factor compared to the existing data [27] in low $Q^{2}$. We find that our prediction is in excellent agreement with the data [27]. We then calculate the kaon form factor up to $Q^{2}=5 \mathrm{GeV}$ to anticipate the higher $Q^{2}$ data which will collect soon [28,29], as in Fig. 2; however, experimentally, the kaon form factor is poorly known.

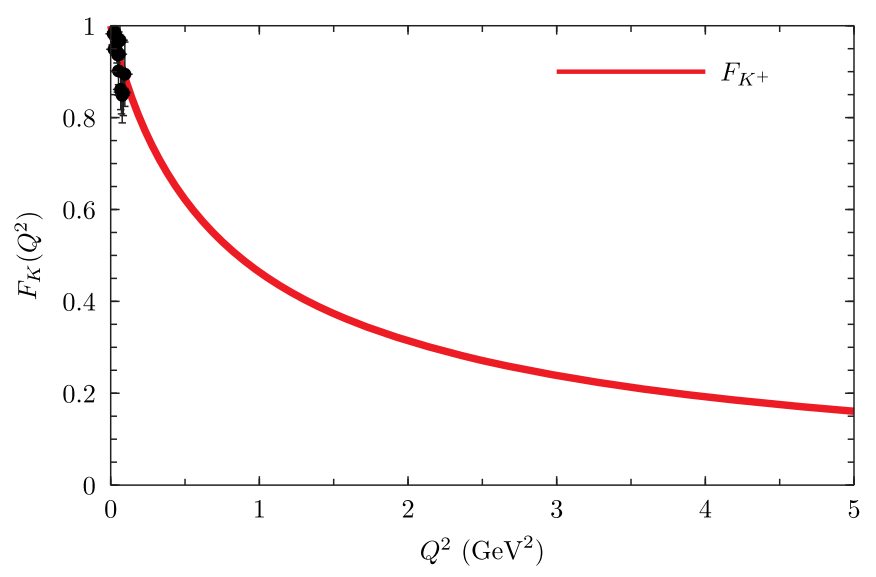

FIG. 2. The kaon form factor (solid line) compared with data at low $Q^{2}$. The experiment data are taken from Ref. [27].

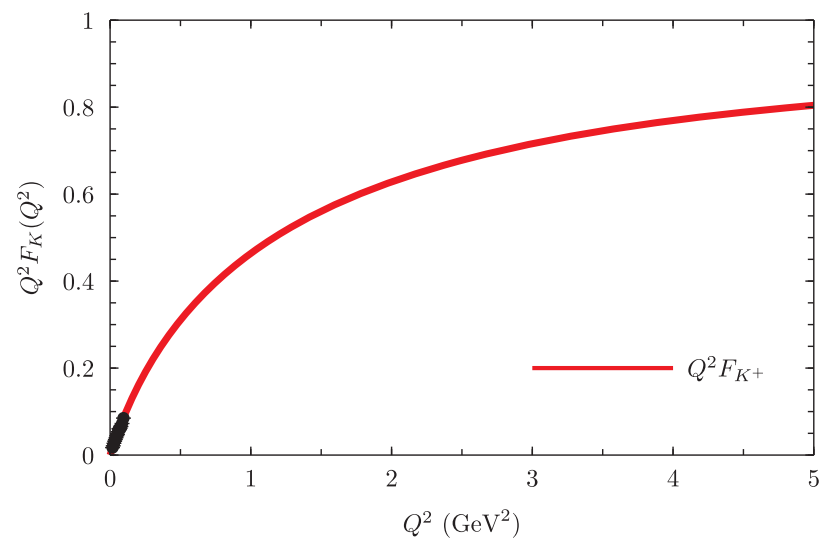

FIG. 3. The same as in Fig. 1 but for $Q^{2} F_{K}^{+}\left(Q^{2}\right)$. The experiment data are taken from Ref. [27].

Figure 3 shows the same results as in Fig. 2, but for $Q^{2} F_{K}\left(Q^{2}\right)$. For larger $Q^{2}$ (asymptotic region), the bulk-toboundary propagator is written as

$$
V^{a}\left(Q^{2}, z\right) \stackrel{Q^{2} \rightarrow \infty}{=}(Q z) K_{1}(Q z) \approx \sqrt{\frac{\pi Q z}{2}} e^{-Q z},
$$

which goes to zero unless $z$ is infinitesimal, $z \sim 1 / Q$. Note that the first term in Eq. (54) goes to $g_{5}^{2}\left(f_{n}^{a}\right)^{2}$ when $z \rightarrow 0$, while the second term goes like $\varepsilon^{2} \rightarrow 0$. The quantity $z V^{a}\left(Q^{2}, z\right)$ behaves like a delta function picking up $\rho_{n n}^{a}(z)$ at $z \rightarrow 0$. The upper limit of the form factor integral can be set to infinity as the integrated vanish at large $z$. Then, the kaon form factor in higher $Q^{2}$ is defined by

$$
\begin{aligned}
F^{a}\left(Q^{2}\right) & \stackrel{Q^{2} \rightarrow \infty}{=} \frac{g_{5}^{2}\left(f_{n}^{a}\right)^{2}}{Q^{2}} \int_{0}^{\infty} d w w^{2} K_{1}(w) \\
& =\frac{2 g_{5}^{2}\left(f_{n}^{a}\right)^{2}}{Q^{2}}=\frac{8 \pi^{2}\left(f_{n}^{a}\right)^{2}}{Q^{2}} .
\end{aligned}
$$

We find that the kaon form factor for larger $Q^{2}$ agrees well with the perturbative QCD prediction [31].

Using Eq. (56), we obtain the charge radius for the lightest kaon $r_{K^{+}}=0.56 \mathrm{fm}$. We find that our result is in excellent agreement with the experimental data [27] and PDG [32].

We also compare our model approach with the work of Ref. [33], which uses a light-front (LF) holographic approach, where the holographic expression in 5D AdS space is matched to QCD in the LF frame. In this approach, to incorporate the quark mass, the "effective potential" in the AdS space is modified by adding a term to obtain the meson mass expression matches with the quark mass contribution in LF QCD. Contrary to this approach, we introduce the quark mass parameter, as a source of the quark bilinear operator $\bar{\psi}_{R} \psi_{L}$ in the AdS boundary, which is consistent with the AdS/CFT rule, and it appears as a 
coefficient in the background field, $X_{0}$. Consequently, the quark mass parameter appears differently in the effective potential, compared to the work of Ref. [33]. In addition, they identify the light-front wave function, where the hadron properties are encoded, by comparing the electromagnetic form factor in AdS and the LF QCD form factor.

In comparing our obtained results with their results on the kaon form factors, their results for the charge radius of the $K^{+}$are slightly larger than our result in the low $Q^{2}$ regime, where the charge radius is $0.615 \mathrm{fm}$ for the dynamical spin parameter $B=0$ and even larger for $B>0$. However, the behavior prediction of the kaon form factor in the large $Q^{2}$ regime, which goes like $1 / Q^{2}$, is similar to our obtained result.

We note that, in this paper, we started with an AdS Lagrangian that has $S U(3)_{L} \times S U(3)_{R}$ symmetry, and it reproduces a chiral symmetry breaking of QCD. An approximate relation due to a chiral-symmetry-breakinglike, Gell-Mann-Oakes-Renner relation is preserved in our approach.

\section{SUMMARY}

In summary, we have computed the kaon form factor in holographic QCD, which is a complementary approach of QCD. We adopt a bottom-up approach of the AdS/CFT correspondence, instead of a top-down approach, where we employ the properties of QCD to construct its 5D gravity dual theory. We begin to describe the AdS/CFT correspondence formalism, describing a correspondence between 4D operators $\mathcal{O}(x)$ and fields in the 5D bulk $\phi(x, z)$. We calculate the kaon form factor in holographic QCD.

The result for the kaon form factor is in good agreement with the existing data in low $Q^{2}$. We then predict the kaon form factor in higher $Q^{2}$. We found that the kaon form factor in higher $Q^{2}$ is consistent with the perturbation QCD prediction.

We finally calculate the charge radius of the kaon in holography QCD. We obtained $r_{K}^{+}=0.56 \mathrm{fm}$, which is in excellent agreement with the data as well as the Particle Data Group. In the future, it would be interesting to extend the calculation of the form factor and gravitational form factor of the $\mathrm{B}$ and $\mathrm{D}$ mesons, which contain the bottom and charm quarks, respectively, using the holographic QCD model.

\section{ACKNOWLEDGMENTS}

The work of P. T. P. H. was supported by the Ministry of Science, Information and Communication Technology (ICT) and Future Planning, Gyeongsangbuk-do and Pohang City through the Young Scientist Training AsiaPacific Economic Cooperation program of Asia Pacific Center for Theoretical Physics (APCTP).
[1] C. G. Callan, Jr., R. F. Dashen, and D. J. Gross, Toward a theory of the strong interactions, Phys. Rev. D 17, 2717 (1978).

[2] W. J. Marciano and H. Pagels, Quantum chromodynamics: A review, Phys. Rep. 36, 137 (1978).

[3] P. T. P. Hutauruk, I. C. Cloet, and A. W. Thomas, Flavor dependence of the pion and kaon form factors and parton distribution functions, Phys. Rev. C 94, 035201 (2016).

[4] W. W. Buck, R. A. Williams, and H. Ito, Elastic charge form-factors for $K$ mesons, Phys. Lett. B 351, 24 (1995).

[5] P. C. Tandy, Hadron physics from the global color model of QCD, Prog. Part. Nucl. Phys. 39, 117 (1997).

[6] E. O. da Silva, J. P. B. C. de Melo, B. El-Bennich, and V. S. Filho, Pion and kaon elastic form factors in a refined lightfront model, Phys. Rev. C 86, 038202 (2012).

[7] A. F. Krutov, S. V. Troitsky, and V. E. Troitsky, The $K$ meson form factor and charge radius: Linking low-energy data to future Jefferson Laboratory measurements, Eur. Phys. J. C 77, 464 (2017).

[8] J. Koponen, A. Zimermmane-Santos, C. Davies, G. P. Lepage, and A. Lytle, Light meson form factors at high $Q^{2}$ from lattice QCD, EPJ Web Conf. 175, 06015 (2018).
[9] H. J. Kwee and R. F. Lebed, Pion form factor in improved holographic QCD backgrounds, Phys. Rev. D 77, 115007 (2008).

[10] H. R. Grigoryan and A. V. Radyushkin, Pion form-factor in chiral limit of hard-wall AdS/QCD model, Phys. Rev. D 76, 115007 (2007).

[11] H. R. Grigoryan and A. V. Radyushkin, Structure of vector mesons in holographic model with linear confinement, Phys. Rev. D 76, 095007 (2007).

[12] J. Babington, J. Erdmenger, N. J. Evans, Z. Guralnik, and I. Kirsch, Chiral symmetry breaking and pions in nonsupersymmetric gauge / gravity duals, Phys. Rev. D 69, 066007 (2004).

[13] Z. Abidin and C. E. Carlson, Gravitational form factors of vector mesons in an AdS/QCD model, Phys. Rev. D 77, 095007 (2008).

[14] T. Gutsche, V. E. Lyubovitskij, I. Schmidt, and A. Vega, Dilaton in a soft-wall holographic approach to mesons and baryons, Phys. Rev. D 85, 076003 (2012).

[15] A. Ballon-Bayona, G. Krein, and C. Miller, Strong couplings and form factors of charmed mesons in holographic QCD, Phys. Rev. D 96, 014017 (2017). 
[16] G. F. de Teramond and S. J. Brodsky, Hadronic Spectrum of a Holographic Dual of QCD, Phys. Rev. Lett. 94, 201601 (2005).

[17] T. Sakai and J. Sonnenschein, Probing flavored mesons of confining gauge theories by supergravity, J. High Energy Phys. 09 (2003) 047.

[18] A. M. Polyakov, String theory and quark confinement, Nucl. Phys. B, Proc. Suppl. 68, 1 (1998).

[19] T. Gherghetta, J. I. Kapusta, and T. M. Kelley, Chiral symmetry breaking in the soft-wall AdS/QCD model, Phys. Rev. D 79, 076003 (2009).

[20] T. Sakai and S. Sugimoto, Low energy hadron physics in holographic QCD, Prog. Theor. Phys. 113, 843 (2005).

[21] K. Ghoroku and M. Yahiro, Chiral symmetry breaking driven by dilaton, Phys. Lett. B 604, 235 (2004).

[22] J. M. Maldacena, The large $N$ limit of superconformal field theories and supergravity, Int. J. Theor. Phys. 38, 1113 (1999); Adv. Theor. Math. Phys. 2, 231 (1998).

[23] E. Witten, Anti-de Sitter space and holography, Adv. Theor. Math. Phys. 2, 253 (1998).

[24] J. Erlich, E. Katz, D. T. Son, and M. A. Stephanov, QCD and a Holographic Model of Hadrons, Phys. Rev. Lett. 95, 261602 (2005).
[25] L. Da Rold and A. Pomarol, Chiral symmetry breaking from five dimensional spaces, Nucl. Phys. B721, 79 (2005).

[26] S. J. Brodsky and G. F. de Teramond, Hadronic Spectra and Light-Front Wavefunctions in Holographic QCD, Phys. Rev. Lett. 96, 201601 (2006).

[27] S. R. Amendolia et al., A measurement of the kaon charge radius, Phys. Lett. B 178, 435 (1986).

[28] M. Carmignotto et al., Separated kaon electroproduction cross section and the kaon form factor from $6 \mathrm{GeV}$ JLab data, Phys. Rev. C 97, 025204 (2018).

[29] T. Horn, Meson form factors and deep exclusive meson production experiments, EPJ Web Conf. 137, 05005 (2017).

[30] Z. Abidin and C. E. Carlson, Strange hadrons and kaon-topion transition form factors from holography, Phys. Rev. D 80, 115010 (2009).

[31] G. P. Lepage and S. J. Brodsky, Exclusive processes in perturbative quantum chromodynamics, Phys. Rev. D 22, 2157 (1980).

[32] K. A. Olive et al. (Particle Data Group), Review of particle physics, Chin. Phys. C 38, 090001 (2014).

[33] M. Ahmady, C. Mondal, and R. Sandapen, Dynamical spin effects in the holographic light-front wavefunctions of light pseudoscalar mesons, Phys. Rev. D 98, 034010 (2018). 